\title{
The Jeep Problem: A Flexible MIP Formulation
}

\author{
Uwe Gotzes $^{1}$, Claudia Gotzes ${ }^{1}$ \\ ${ }^{1}$ University of Duisburg-Essen, Thea-Leymann-Straße 9, 45127 Essen, Germany \\ Correspondence to: Uwe Gotzes, e-mail: uwe.gotzes@uni-due.de
}

\begin{abstract}
This article deals with the Jeep Problem (also known as Desert Crossing Problem), which reads as follows: An unlimited supply of fuel is available at one edge of a desert, but there is no source on the desert itself. A vehicle can carry enough fuel to go a certain distance, and it can built up its own refuelling stations. What is the minimum amount of fuel the vehicle will require in order to cross the desert? Under these mild conditions this question is answered since the 1940s. But what is the answer if the caches are restricted to certain areas or if the fuel consumption does not depend linearly on the distance travelled? To answer these and similar questions we develop and solve a flexible mixed-integer programming (MIP) model for the classical problem and enhance it with new further aspects of practical relevance.
\end{abstract}

Keywords: Jeep problem, Crossing the desert, Logistics, Storage, Continuous location, Mixed-integer programming

\section{Introduction}

This article was motivated by the reading of the chapter Desert Crossing Problem in Martin Gardner's book My Best Mathematical and Logic Puzzles [7, pp. 12, 54]. We wondered if the described problem (see abstract) can also be solved with mixed-integer programming techniques. Analytical solutions for the classical problem are available since the 1940s [1, 5, 14. If, for instance, the topography requires that the caches are restricted to certain areas or if it is desired that the fuel consumption is modelled more accurate than just by a linear relationship, analytical solutions are not, at least not yet available.

For instance the operator of a rescue helicopter in the Himalayas can be faced with those tricky circumstances [13, pp. 231ff.], [2, pp. 1286ff.].

In section 2 we provide all sets, parameters and variables that we will need to set up the optimisation models that solve the outlined and similar problems. In section 3 we built up the basic model to solve the classical problem and in section 4 we extend and modify the basic model such that it is capable to answer the other questions. In section 5 we give brief examples of numerical solutions, followed by the concluding section 6 .

\section{Parameters, Sets and Variables}

The tables 1 and 2 contain the parameters, sets and variables to set up the basic mixed-integer programming model for the classical problem and for its enhanced versions that incorporate the modelling of forbidden zones and complex fuel consumption patterns. In forbidden zones it is not possible to open a fuel depot, for instance due to lake areas, steep mountains or insecure conditions. Amongst others, fuel consumption can depend on the altitude or the ground conditions.

\section{The Basic Model}

As we want to minimise the total fuel consumption of the vehicle, our objective is to minimise $F$. To break the massive symmetry due to possible but unnecessary depots and moving options in the model we add a 
Table 2: Variables that are used in the model for the classical problem and its enhanced versions.

\begin{tabular}{|l|l|}
\hline \multicolumn{2}{|c|}{ Variables } \\
\hline \hline basic model & \\
\hline$f_{t} \geq 0, t \in T$ & fuel in tank after move $t$ \\
$w_{i} \geq 0, i \in V \backslash\{0\}$ & length of route segment $i$ between depot $i-1$ and $i$ \\
$W_{i} \geq 0, i \in V$ & auxiliary variables: end of segment $i$ \\
$g_{i, t} \in\{0,1\},(i, t) \in(V \backslash\{0\}) \times T$ & use edge $i$ in step $t$ ? $\left(g_{i, t}=1 \widehat{=}\right.$ true, $g_{i, t}=0 \widehat{=}$ false) \\
$a_{i, t} \in\{0,1\},(i, t) \in V \times T$ & at position $i$ after move $t$ ? ( $a_{i, t}=1 \widehat{=}$ true, $a_{i, t}=0 \widehat{=}$ false) \\
$\delta_{i, t} \in[-c, c],(i, t) \in V \times T$ & drop fuel at depot or refuel at depot (amount) \\
$l_{i, t} \geq 0,(i, t) \in(V \backslash\{0\}) \times T$ & fuel in depot after dropping or refuelling \\
$p_{i, t} \geq 0,(i, t) \in(V \backslash\{0\}) \times T$ & product of $g_{i, t}$ and $w_{i}$ (equates to the fuel consumption while using \\
& role of $w_{i}$ \\
$F \geq 0$ & total fuel consumption \\
\hline forbidden zones model & \\
\hline$r_{i, j} \in\{0,1\}, i \in(V \backslash\{0\}) \times\{1, \ldots, m\}$ & auxiliary variables to model forbidden zones \\
\hline complex fuel consumption model & \multicolumn{2}{|c|}{ auxiliary variables to model the piecewise linear function $f$} \\
\hline$\lambda_{i, k} \in[0,1],(i, k) \in V \times K$ & auxiliary variables to model the piecewise linear function $f$ \\
$y_{i, k} \in\{0,1\},(i, k) \in V \times K \backslash\{\kappa\}$ & integral of $s$ from base to depot $i$ \\
$I_{i} \geq 0, i \in V$ &
\end{tabular}

The vehicle either moves between neighbouring depots which is modelled through equations (6) and (7) or it rests at its goal by equation (8):

$$
\begin{aligned}
a_{1, t} & \geq a_{0, t-1}, \forall t \in T \backslash\{0\} \\
a_{i-1, t}+a_{i+1, t} & \geq a_{i, t-1}, \forall(i, t) \in(V \backslash\{0, n\}) \times(T \backslash\{0\}) \\
a_{n-1, t}+a_{n, t} & \geq a_{n, t-1}, \forall t \in T \backslash\{0\} .
\end{aligned}
$$

To capture the vehicle's fuel usage, we have to track if the segment $w_{i}$ between depot $i-1$ and depot $i$ was used during move $t$. For this purpose we use the binary variables $g_{i, t}$ that are subject to the following constraints for all $(i, t) \in S$ :

$$
g_{i, t} \geq \frac{2 \cdot\left(a_{i-1, t}+a_{i, t}\right)-3}{4} \quad \text { and } \quad g_{i, t} \geq \frac{2 \cdot\left(a_{i, t-1}+a_{i-1, t}\right)-3}{4} .
$$

The right hand side of the left equation is greater than 0 iff segment $i$ was travelled in forward direction in step $t$ and the right hand side of the right equation is greater than 0 iff segment $i$ was travelled in backward direction in step $t$. The vehicle's fuel usage on arc $i$ in step $t$ is the product $p_{i, t}$ of the arcs length $w_{i}$ and $g_{i, t}$. To model this product in our MIP formulation, we apply the standard modelling technique, amongst others, presented in [3, pp. 83-84] for all $(i, t) \in S$, yielding

$$
p_{i, t} \leq c \cdot g_{i, t}, \quad p_{i, t} \leq w_{i}, \quad p_{i, t} \geq w_{i}-c \cdot\left(1-g_{i, t}\right) .
$$

Once we have the products $p_{i, t}$ at hand, the fuel in the tank after step $t$ underlies the "fuel constraints". The fuel in the tank after step $t$ equals the fuel in the tank after step $t-1$ minus the fuel consumed to travel to a neighbouring depot plus the fuel exchange between the tank and the respective depot after step $t-1$ :

$$
f_{t}=f_{t-1}-\sum_{i \in V \backslash\{0\}} p_{i, t}+\sum_{i \in V} \delta_{i, t-1}, \forall t \in T \backslash\{0\} .
$$

The "depot constraints" that capture the interaction between depot usage and depot level are:

$$
l_{i, t}=l_{i, t-1}-\delta_{i, t}, \forall(i, t) \in S
$$

The depot level after step $t$ is the depot level after step $t-1$ minus the fuel exchange between the tank and the respective depot. Note that the $\delta_{i, t}$ in equation $(12)$ have the opposite sign as in $(11)$ as filling the tank corresponds to emptying a depot and vice versa. 
To use depot $i$ in step $t$, the vehicle has to be at the depot's location for $(i, t) \in V \times T$, what can be modelled as

$$
\delta_{i, t} \leq c \cdot a_{i, t} \quad \text { and } \quad \delta_{i, t} \geq-c \cdot a_{i, t} .
$$

If the vehicle is at position $i$ after move $t,(13)$ yields $\delta_{i, t} \in[-c, c]$. Otherwise $a_{i, t}=0$ and hence $\delta_{i, t}=0$.

We assume that, at the beginning of the journey, the vehicle rests at the edge of the desert and that its tank and all depots are empty, i.e.

$$
a_{0,0}=0, \quad f_{0}=0 \quad \text { and } \quad l_{i, 0}=0, \forall i \in V .
$$

At this point, it is clear, that our model is flexible enough to easily implement other assumptions with practical relevance. For instance, simply set $l_{i, 0}$ to a desired value, to model that depot $i$ is not empty in the beginning.

Finally it is necessary to require that the crossing really has to be done. That is, the total length of all path segments $w_{i}$ has to be the total distance $d$ :

$$
\sum_{i \in V \backslash\{0\}} w_{i}=d
$$

Remember that $p_{i, t}$ is the fuel consumption when segment $i$ is crossed in step $t$. For convenience we use the variable $F$ to track the total fuel usage:

$$
F=\sum_{(i, t) \in S} p_{i, t}
$$

\section{Model Extensions and Variations}

In this section we come to the announced model extensions and variations, that emphasise the flexibility of our approach.

\subsection{Model extension: Forbidden zones}

With the basic model at hand, it is quite simple to incorporate spatial restrictions for depots. For every $(i, j) \in(V \backslash\{0\}) \times\{1, \ldots, m\}$ just add

$$
W_{i}-\underline{d_{j}} \leq d \cdot r_{i, j} \quad \text { and } \quad W_{i}-\overline{d_{j}} \geq-d \cdot\left(1-r_{i, j}\right)
$$

to the model. If $W_{i} \leq d_{j}$ or $W_{i} \geq \overline{d_{j}}$, 17) can easily be fulfilled by the right choice for $r_{i, j}$. On the other hand, if $W_{i} \in\left[\underline{d_{j}}, \overline{d_{j}}\right]$, no $r_{i, j}$ exists such that both parts of (17) hold.

\subsection{Model extension: Position dependent fuel consumption}

To model position dependent fuel costs, for every $i \in V$, add the following constraints to the model:

$$
\begin{array}{r}
W_{i}=\sum_{k \in K} \lambda_{i, k} \cdot x_{k}, \quad \sum_{k \in K} \lambda_{i, k}=1, \quad \sum_{k \in K \backslash \kappa} y_{i, k}=1 \\
\lambda_{i, 1} \leq y_{i, 1} \quad, \quad \lambda_{i, k} \leq y_{i, k-1}+y_{i, k}, \quad \forall k \in K \backslash\{1, \kappa\} \quad, \quad \lambda_{i, \kappa} \leq y_{i, \kappa-1} \\
I_{i}=\sum_{k \in K} \lambda_{i, k} \cdot f\left(x_{k}\right) .
\end{array}
$$

This is a standard technique to model piecewise linear functions in MIP models 4. The idea behind it is to express argument and function value as convex combinations of interval bounds of the function's linearity 
regions and of the function values at the interval bounds, respectively. The $y$-variables "identify" the relevant linearity region and ensure that at most the two associated neighbouring $\lambda$-variables are positive. For instance, if $y_{i, k}=1$, then only $\lambda_{i, k}$ and $\lambda_{i, k+1}$ can be different from zero and $\lambda_{i, k}+\lambda_{i, k+1}=1$. The $\lambda$-variables are the coefficients for the convex combinations. We need the $I_{i}$ from equation 20 to complete the model and the equations in (18) and $(19)$ to make the $I_{i}$ take on the desired values $f\left(W_{i}\right)$ (see tables 1 and 2 . For every $(i, t) \in S$ we replace the constraints 10 by

$$
p_{i, t} \leq M \cdot g_{i, t}, \quad p_{i, t} \leq\left(I_{i}-I_{i-1}\right), \quad p_{i, t} \geq\left(I_{i}-I_{i-1}\right)-M \cdot\left(1-g_{i, t}\right) .
$$

The $p_{i, t}$ variables then take on the value $\int_{W_{i-1}}^{W_{i}} s(x) \mathrm{d} x=I_{i}-I_{i-1}$ as desired.

\subsection{Model variation: Exploration problem}

If the vehicle is supposed to return to the base after it has crossed the desert, this can be modelled by simply adding the constraint $a_{0, \tau}=1$.

\subsection{Model variation: Transport fuel across the desert}

A related problem arises if we pose the question how much fuel can be transported across the desert if there is only a limited amount $B$ of fuel available at the base? To derive a suitable model for this question from the preceding models, it is sufficient to alter the objective according to maximise $l_{n, \tau}$. Furthermore a few additional constraints are required, namely $F \leq B$ and $-\delta_{n, \tau} \leq f(\tau)$.

\subsection{Model variation: Maximise distance}

To answer the question "How far can the vehicle travel with a given amount of fuel at the base?", the objective has to be modified to maximise $d$, where $d$ now is a variable and no longer a parameter as in the other models and variants. Additionally it is necessary to limit the available fuel, i.e. $F \leq B$.

\section{Computational Results}

In this section we provide a brief discussion of numerical experiments for the MIP formulation of the classical problem and its two extensions and also for the three variants presented in this text. All computations were done on a standard Linux PC with an Intel@ Core $^{\mathrm{TM}}$ i5-3570 CPU @ $3.40 \mathrm{GHz}$ and 16 GB RAM. To translate the mathematical models into mixed-integer programs expressed in the .lp file format which can be read by a MIP solver we used Zimpl [12]. As MIP solver we applied Gurobi 7.0.2 [9].

\subsection{Computational results for the basic model}

Exemplarily, we choose $c=1, d=1+1 / 3+1 / 5+1 / 7, n=3$ and $\tau=15$. The optimiser finds an optimal solution with $F \approx 5.76$, while 4 is the optimal solution [14. The reason for this is, that by the choice of $n$ and $\tau$, we overly restricted the search space. If we choose $n>3$ and $\tau>15$, the optimal value of 4 with dumps at $w_{i}=\sum_{j=1}^{i} 1 /(2 j+1), i=1,2,3$, requiring 16 movements between caches is found. We call such a movement a trip.

\subsection{Computational results for the model "Forbidden zones"}

Now let us choose $\left[1 / 14,1 / 7+1 /{ }_{10}\right]$ as a restricted area. In this case the optimal caches are the same as for the basic model, except the first one at $1 / 7$ is replaced by two caches. One at $2857 . \times 10^{-5}$ and one at $1 / 7+1 / 10$. 
The amount of fuel that is necessary is $F \approx 4.257$. Stretching the restricted area to $[1 / 14,1 / 7+1 / 5+1 / 14]$ yields $F \approx 5.629$. In the first case 25 trips are needed, while in the latter only 24 are required to achieve the optimum.

\subsection{Computational results for the model "Position dependent fuel consump- tion"}

We choose $\left(x_{k}, f\left(x_{k}\right)\right)_{k=1}^{5}$ defining the piecewise linear function $f$ as shown in table 3 . For $n=4, \tau=30$ the minimum fuel amount for the crossing is $F \approx 6.92$ and 24 trips are required.

\begin{tabular}{|l|l|l|}
\hline$k$ & $x_{k}$ & $f\left(x_{k}\right)$ \\
\hline 1 & 0 & 0 \\
2 & $1 / 7$ & $0.5 \cdot x_{2}$ \\
3 & $1 / 7+1 / 5$ & $0.6 \cdot x_{3}$ \\
4 & $1 / 7+1 / 5+1 / 3$ & $0.75 \cdot x_{4}$ \\
5 & $1 / 7+1 / 5+1 / 3+1$ & $1.1 \cdot x_{5}$ \\
\hline
\end{tabular}

Table 3: Characterisation of the piecewise-linear function $f$

\subsection{Computational results for the "Exploration variant"}

Let us choose $c=1, d=1 / 2+1 / 4+1 / 6+1 / 8, n=6$ and $\tau=40$. The optimiser finds an optimal solution with $F=3$ and dumps at $w_{i}=\sum_{j=1}^{i} 1 /(2 j), i=1,2,3,4$, requiring 20 trips (cf. [14]) after $100 \mathrm{~s}$.

\subsection{Computational results for the "Maximise fuel transport variant"}

We set $n=5, \tau=20, d=c=1$ and $B=3$ and wonder how much of the 3 units fuel can be transported across the distance? The answer is $l_{n, \tau}=0.5 \overline{3}$. Nine trips $(0 \rightarrow 1 \rightarrow 0 \rightarrow 1 \rightarrow 0 \rightarrow 1 \rightarrow 2 \rightarrow 1 \rightarrow 2 \rightarrow 3)$ and two caches along the way, located at $1 / 5$ an $1 / 3$, are needed.

\subsection{Computational results for the "Maximise distance variant"}

A solution which yields the minimum amount of gasoline for a given distance is also a solution which yields the maximum distance for a given amount, and conversely [14]. From a theoretical perspective it so happens, that a solution for the latter conditions appears much easier to obtain [14]. This statement only holds in a very limited sense for the MIP models. We choose $n=7, \tau=30$ and $c=1$ in both models, $d=1+1 / 3+1 / 5+1 / 7$ in the basic model and $B=4$ (which is the optimal fuel consumption in the basic model) in the "maximise distance" model. We used Gurobi to solve both models and it turned out that the maximum distance variant was solved significantly faster (430 s) than the basic model (640 s). As a second test we used Gurobi's tune feature with a time limit of 24 hours for the basic model and also for the maximise distance variant. With the improved parameter settings suggested by the tuner, both models could be solved in more or less the same period of time (210 s versus $205 \mathrm{~s})$. The four times mentioned in this subsection are all average times over ten runs with different random seeds.

\section{Conclusion}

Many readers called attention to the previously published discussions of the problem and hundreds of letters were received, giving general solutions and interesting sidelights [7, (cf. [1, 15, 6, 10, 11, 14, 15, and the references therein). With our MIP formulation we add a new facet to the topic. The model is 
Journal of Nepal Mathematical Society (JNMS), Vol. 1, Issue 1 (2018); U. Gotzes, C. Gotzes

flexible enough to be ready for further extensions like direction dependent fuel costs or additional "fix costs" in terms of fuel consumption for the usage of a depot (landing and launching a helicopter is extra costly). However some important questions remain unanswered. Do elegant analytical solutions exist for the presented problems? How large have $n$ and $\tau$ to be chosen in the different models? On the other hand it is also a feature of the models that they can answer questions like "What is the optimum with at most $n$ depots and $\tau$ movements?". Finally, note that all models discussed in this article are combinable. The Zimpl files [12] of the problems are made available under [8].

\section{References}

[1] Alway GG (1957) Crossing the desert. The Mathematical Gazette 41(337), 209.

[2] Auerbach PS (2016) Wilderness Medicine E-Book, chap. Helicopter Rescue in the Wilderness, p. 1270. Elsevier Health Sciences. https://books.google.de/books?id=02EgDQAAQBAJ.

[3] Bishop J (2017) AIMMS Optimization Modeling. AIMMS B.V., Diakenhuisweg 29-35, 2033 AP Haarlem, The Netherlands. https://download.aimms.com/aimms/download/manuals/AIMMS3_OM.pdf

[4] Dantzig GB (1960) On the significance of solving linear programming problems with some integer variables. Econometrica 28(1), 30-44.

[5] Fine NJ (1947) The jeep problem. The American Mathematical Monthly 54(1), 24-31. http://www. jstor.org/stable/2304923.

[6] Gale D (1970) The jeep once more or jeeper by the dozen. The American Mathematical Monthly 77(5), 493-501. http://www.jstor.org/stable/2317382.

[7] Gardner M (1994) My Best Mathematical and Logic Puzzles, chap. 25. Crossing the Desert, pp. 12, 54, Dover Publications. https://books.google.de/books?id=sUuBCzazfYUC\&printsec=frontcover

[8] Gotzes U, Gotzes C (2017) The jeep problem-Zimpl files. https://www.uni-due.de/ si203go/ \# jeepproblem.

[9] Gurobi Optimization, Inc. (2016) Gurobi Optimizer Reference Manual. http://www.gurobi.com.

[10] Hausrath A, Jackson B, Mitchem J, Schmeichel E (1995) Gale's round-trip jeep problem. The American Mathematical Monthly 102(4), 299-309. http://www.jstor.org/stable/2974949.

[11] Helmer O (1946) The jeep problem. Problem in Logistics Project Rand, Report No. RA-15015.

[12] Koch T (2004) Rapid mathematical prototyping. Ph.D. thesis, Technische Universität Berlin.

[13] Leishman GJ (2006) Principles of Helicopter Aerodynamics, chap. 5.5.5 Engine Fuel Consumption, pp. 231-233. Cambridge University Press.

[14] Phipps CG (1947) The jeep problem: A more general solution. The American Mathematical Monthly 54(8), 458-462. http: //www.jstor.org/stable/2305704

[15] Weisstein EW (2017) "Jeep Problem." From MathWorld-A Wolfram Web Resource. http:// mathworld.wolfram.com/JeepProblem.html 